\title{
Operationalisierung von Qualitätsindikatoren mittels Routinedaten am Beispiel der Evaluation der „Integrierten Versorgung Gesundes Kinzigtal“‘
}

\section{Operationalization of Quality Indicators with Routine Data Using the Example of the Evaluation of "Integrated Care Healthy Kinzigtal"}

\author{
Autoren \\ Ingrid Köster ${ }^{1}$, Claudia Mehl2 ${ }^{*}$, Achim Siegel ${ }^{3}$, Erika Graf ${ }^{4}$, Dominikus Stelzer ${ }^{4}$, Erik Farin-Glattacker ${ }^{5}$, \\ Max Geraedts ${ }^{2 * *}$, Ingrid Schubert ${ }^{* *}$
}

Institute

1 PMV forschungsgruppe an der Medizinischen Fakultät und Uniklinik Köln, Universität zu Köln, Köln, Deutschland

2 Institut für Versorgungsforschung und Klinische Epidemiologie, Philipps-Universität Marburg, Marburg, Deutschland

3 Institut für Arbeitsmedizin, Sozialmedizin und Versorgungsforschung, Universitätsklinikum Tübingen, Tübingen, Deutschland

4 Institut für Medizinische Biometrie und Statistik, Universitätsklinikum Freiburg, Medizinische Fakultät, Albert-Ludwigs-Universität Freiburg, Freiburg, Deutschland

5 Universitätsklinikum Freiburg, Institut für Medizinische Biometrie und Statistik, Sektion Versorgungsforschung und Rehabilitationsforschung, Freiburg, Deutschland

\section{Schlüsselwörter}

Qualitätsindikatoren, GKV-Routinedaten, Operationalisierung, Gesundheitsversorgungsforschung, Integrierte Versorgung

Key words

Quality Indicators, administrative data, operationalisation, integrated care

online publiziert $\quad 10.11 .2021$

\section{Bibliografie}

Gesundheitswesen 2021; 83 (Suppl. 2): S87-S96

DOI 10.1055/a-1585-1735

ISSN $0949-7013$

(C) 2021. Thieme. All rights reserved.

Georg Thieme Verlag, Rüdigerstraße 14,

70469 Stuttgart, Germany

geteilte Erstautorenschaft,
Korrespondenzadresse

Dr. Ingrid Schubert

PMV forschungsgruppe an der KJP Universität zu Köln

Herderstraße 52

50931 Köln

Deutschland

ingrid.schubert@uk-koeln.de

$\circledast$ Zusätzliches Material finden Sie unter https://doi.org/ 10.1055/a-1585-1735

\section{ZUSAMMENFASSUNG}

Ziel Für die 10-Jahres-Evaluation der Integrierten Versorgung „Gesundes Kinzigtal“ (IVGK, Innovationsfonds-Projekt 01VSF1 6002) konsentierte eine multidisziplinäre Expertengruppe 101 Qualitätsindikatoren (QI), mit denen die Qualität der regional integrierten Versorgung mit ihrer Ausrichtung auf Gesundheits- und Präventionsprogramme evaluiert werden sollte. Ein Kriterium war, dass sich diese QI prinzipiell für eine Abbildung mittels Routinedaten eignen sollten. Ziel der Studie war es, zu prüfen, wie viele der und auf welche Weise die entwickelten QI in Deutschland tatsächlich mit Routinedaten abgebildet werden können und aus welchen Gründen eine Operationalisierung eingeschränkt oder nicht möglich war.

Material und Methode Die Operationalisierung der QI erfolgte mittels pseudonymisierten Abrechnungsdaten der AOK Baden-Württemberg der Jahre 2006-2015, die das Wissenschaftliche Institut der AOK (WIdO) dem Evaluationsteam zur Verfügung stellte. Alle operationalisierten Indikatoren waren binär kodiert (Kriterium erfüllt ja/nein). Die in den Zähler- und Nennerdefinitionen benannten Diagnosen, Prozeduren oder Arzneimittel wurden über ICD-10-Kodes (Ein- und Ausschlussdiagnosen), EBM-Kennziffern, OPS-Schlüssel und ATC-Kodes operationalisiert. Indikatorprävalenzen wurden im zeitlichen Verlauf zur Prüfung von Auffälligkeiten als Hinweis auf mögliche Fehlkodierung untersucht.

\footnotetext{
** geteilte Letztautorenschaft
} 
Ergebnisse 90 der 101 Indikatoren waren mit Routinedaten operationalisierbar. 14 der 90 Indikatoren konnten nur mit Einschränkungen operationalisiert werden, da entsprechende Leistungsziffern erst im Beobachtungszeitraum eingeführt oder bestehende Ziffern verändert wurden. 76 der 90 Indikatoren konnten uneingeschränkt operationalisiert werden, davon waren bei 15 Indikatoren Vor- und Nachbeobachtungszeiten notwendig, wodurch sie nicht für alle Jahre dargestellt werden konnten. 11 von 101 QI waren nicht operationalisierbar, da EBM-Ziffern erst nach 2015 eingeführt oder nicht als Einzelleistung für alle Arztgruppen erfasst wurden (z. B. Spirometrie und Langzeit-EKG). Auffällige Verläufe in den Indikatorprävalenzen waren erklärbar.

Schlussfolgerung Routinedaten ermöglichen ein ressourcensparendes Qualitätsmonitoring. Eine Veränderung der Datengrundlage im Beobachtungszeitraum, wie etwa durch Neueinführung oder Streichung von Abrechnungsziffern, erschwert die longitudinale, routinedatenbasierte Qualitätsbewertung, ermöglicht aber ggfs. für spätere Zeiträume die Operationalisierung weiterer oder neuer Indikatoren.

\section{ABSTRACT}

Aim As part of the 10-year evaluation of Gesundes Kinzigtal Integrated Care (IVGK, Innovation Fund Project 01VSF16002), a multidisciplinary group of experts agreed on 101 quality indicators (QI) to evaluate the quality of regionally integrated care with its focus on health and prevention programs. One criterion was that the selected QI should in principle be suitable for mapping using routine data. The aim of the study was to investigate how many and in what way the QI developed can actually be mapped in Germany with routine data and for what reasons operationalization was restricted or not possible.

Material and methods The operationalization of the QIs was performed using pseudonymized billing data of the AOK Baden-Württemberg from 2006 to 2015, which the Scientific Institute of the AOK (WIdO) provided to the evaluation team. All operationalized indicators were binary coded (criterion fulfilled yes/no). The diagnoses, procedures, or drugs named in the numerator and denominator definitions were operationalized using ICD-10 codes (inclusion and exclusion diagnoses), EBM codes, OPS codes, ATC codes. Indicator prevalences were examined over time to check for abnormalities as an indication of possible misscoding.

Results Ninety of the 101 indicators were operationalizable with routine data. Fourteen of the 90 indicators could only be operationalized with restrictions, as corresponding service codes were only introduced or existing codes were changed during the observation period. Seventy-six of 90 indicators could be operationalized without restrictions. In this context, 15 of these 76 indicators required pre- and follow-up periods, which meant that they could not be presented for all years. Eleven of 101 Qls could not be operationalized because EBM codes were only introduced after 2015 or were not recorded as individual services for all physician groups (e. g., spirometry and long-term ECG). Striking trends in indicator prevalences could be explained.

Conclusion Routine data enable resource-saving quality monitoring. A change in the data basis during the observation period, for example through the introduction or deletion of billing codes, makes the longitudinal, routine data-based quality assessment more difficult, but enables further or new indicators to be operationalized for later periods.

\section{Einleitung}

Qualitätsindikatoren (QI) werden in Deutschland wie auch international eingesetzt, um die Qualität einzelner Leistungserbringer, ganzer Gesundheitssysteme oder die gesundheitliche Lage im Allgemeinen zu bewerten [1-5]. Eigenständige Datenerhebungen, die nur der Qualitätsbewertung dienen, sind aufwändig. Daher werden international Routinedaten präferiert, die entweder Krankenakten entnommen oder aus Abrechnungsdaten generiert werden. Der geringste Zusatzaufwand entsteht bei der Nutzung von Abrechnungsdaten, da diese elektronisch vorliegen. Deren Nachteil besteht darin, dass zum einen diagnostische und therapeutische Leistungen bei Erkrankungen fokussiert und präventive oder palliative Versorgungselemente in geringem Umfang abgebildet werden. Zum anderen werden zumeist nur wenige klinische Daten erfasst, sodass z. B. eine umfassende Risikoadjustierung nur eingeschränkt möglich ist. Letztlich muss zwischen Aufwand und Breite bzw. Tiefe der Qualitätsbewertung abgewogen werden. In Deutschland wurden zum Zweck der Qualitätssicherung seit Anfang der 1970er Jahre typischerweise mit großem Aufwand eigenständige Daten erhoben [6]. In den letzten Jahren werden zunehmend auch
Krankenkassendaten verwendet. Seit 2009 halten Routinedaten zur Qualitätsmessung sukzessive Einzug, auch bei der jetzt unter Verantwortung des Instituts für Qualitätssicherung und Transparenz im Gesundheitswesen (IQTIG) stehenden einrichtungs- und sektorenübergreifenden Qualitätssicherung nach §137a SGB V. Auch die krankenhausvergleichenden Bewertungen elektiver Eingriffe wie sie im Rahmen des AOK-Krankenhausnavigators [7, 8] oder von der Initiative Qualitätsmedizin (IQM) [9] seit Jahren durchgeführt werden, nutzen Routinedaten. Im ambulanten Sektor geht die routinemäßige Nutzung von Krankenkassendaten zur Darstellung von Transparenz und Qualität bis in die frühen 1980er Jahre zurück, beginnend mit der Analyse von Arzneimittelverbrauchsmengen, wie sie bis heute im GKV-Arzneimittelindex $[10,11]$ ausgewiesen werden. Im nächsten Schritt wurden - auch international - Indikatoren zur Beschreibung der Prozessqualität (Verordnungsweise, Diagnostik, Therapie) wie auch zur Darstellung der Ergebnisse der Versorgung (z. B. Mortalität, stationäre Wiederaufnahme) $[12,13]$ für unterschiedliche Patientengruppen und Settings entwickelt [14-16]. In Deutschland werden diese Indikatoren u. a. im Rahmen von Pharmakotherapieberatungen (z. B. PharmPro 
[17]), in der Qualitätszirkelarbeit, zur Evaluation der Umsetzung von Leitlinien $[18,19]$ oder anderen Handlungsempfehlungen, aber auch im Kontext der Disease-Management-Programme oder der hausarztzentrierten Versorgung [20] erhoben und teilweise an die Leistungserbringer zurückgespiegelt. Einige Krankenkassen entwickelten insbesondere für ein Monitoring und für Vergleiche von Arztnetzen eigene Indikatorensets wie BrAvO (Benchmarking regionale Arztnetz-Versorgung) [21] oder QuATRo („Qualität in Arztnetzen - Transparenz mit Routinedaten“) [22].

Der Nachteil der bisher etablierten Indikatorensets besteht darin, dass sie keine umfassende Evaluation der regionalen Gesundheitsversorgung erlauben. Hierzu ist es nötig, ein breites Spektrum an gesundheitsbezogener Leistungserbringung durch ärztliche und andere therapeutische Berufsgruppen in den verschiedenen Sektoren und Settings (ambulante Versorgung mit Prävention, Akutbehandlung und Behandlung chronisch Kranker, Krankenhaus-/ Notfallversorgung, Rehabilitation, Pflege, palliative Versorgung) abzubilden. Ebenso sind Indikatoren, die Ergebnisqualität erfassen, notwendig [23]. Ein solches umfassendes Indikatorenset wurde zur Evaluation der Integrierten Versorgung „Gesundes Kinzigtal“ (IVGK) im Rahmen des INTEGRAL-Projektes (10-Jahres-Evaluation der populationsbezogenen integrierten Versorgung Gesundes Kinzigtal in Aufbau- und Konsolidierungsphase; gefördert vom Gemeinsamen Bundesausschuss, Förderkennzeichen 01VSF16002_ INTEGRAL) systematisch entwickelt und von einer multidisziplinär zusammengesetzten Expertengruppe in einem Delphi-Prozess konsentiert [24]. Die Hauptergebnisse der Evaluation Gesundes Kinzigtal sind kürzlich von Schubert et al. [25] publiziert worden. Bei der Entwicklung des Indikatorensets wurde darauf geachtet, dass die Indikatoren möglichst auf der Basis von Abrechnungsdaten der Krankenkassen berechenbar sein sollten. Ob jedoch die entwickelten Indikatoren in der Praxis tatsächlich mithilfe dieser Daten operationalisiert werden können, war unklar. Diese Prüfung erfolgte im Rahmen der Evaluation nach der Erstellung des Indikatorensets und wird in diesem Beitrag dargestellt: Zu klären waren hierbei die folgenden Fragen:

1. Wie lassen sich die Zähler mit den Indikatorkriterien und die Nenner der Indikatoren mit der jeweiligen relevanten Bezugspopulation mittels der bei den Krankenkassen vorhandenen Daten operationalisieren?

2. Welcher Anteil der Indikatoren, die prinzipiell für eine Analyse mittels Routinedaten geeignet scheinen, kann mit Routinedaten abgebildet werden? Welche Gründe führen dazu, dass ein Indikator nur eingeschränkt oder nicht abgebildet werden kann?

3. Welche Fallstricke sind bei der Operationalisierung zu beachten und welche Besonderheiten zu beobachten?

Der Beitrag ergänzt die Ausführungen von Geraedts et al. [24] zur Entwicklung und Konsentierung der Indikatoren und beantwortet grundlegende Fragen bei der Verwendung von Routinedaten in der Gesundheitsversorgungsforschung.

\section{Material und Methode}

\section{Datenbasis und Variablen}

Ausgangspunkt für die Operationalisierung der Indikatoren war ein konsentiertes tabellarisches Indikatorenset mit 101 Indikatoren für 19 Tracer bzw. Kategorien [24]. Alle operationalisierten Indikatoren waren binär kodiert (Kriterium erfüllt ja/nein). Das Indikatorenset umfasste das Indikator-Statement in Kurz- und Langversion, den Zähler und den Nenner des Indikators, ausgedrückt in Worten sowie erste Hinweise auf Variablen zur Abbildung des Indikators mit Routinedaten. Die hierzu notwendigen Daten der AOK BadenWürttemberg, die für die Evaluation des INTEGRAL-Vorhabens herangezogen wurden, stellte das Wissenschaftliche Institut der AOK (WIdO) für die Jahre 2006-2015 zur Verfügung. Es lagen Angaben vor unter anderem zu Versichertenstammdaten sowie Leistungsdaten aus den Sektoren Krankenhaus und Rehabilitation, vertragsärztliche Versorgung, Arzneiverordnungen (Actrapid), Heilmittel (HIS) (Physiotherapie, Ergotherapie, Logotherapie usw.) und Arbeitsunfähigkeitsmeldungen (AU). Daten zu Hilfsmitteln standen nicht zur Verfügung. Die Leistungsdaten lagen versicherten- und leistungserbringerbezogen mit Angabe eines Kalenderdatums (ambulante und stationäre Leistungen und Verordnungen), eines Quartals (ambulante Diagnosen) oder eines Zeitraums (stationäre Aufenthalte und Diagnosen, $A U$ ) vor. Bei den kalenderjahresbezogenen Analysen wurden in Abhängigkeit von den einbezogenen Informationen jeweils Daten berücksichtigt, deren zugehöriges Abrechnungs- oder Verordnungsdatum oder Quartal in dem Kalenderjahr oder deren Behandlungszeitraum (mindestens teilweise) im Kalenderjahr lag. Angaben der Versichertennummer und des Leistungserbringers (Arzt, Institution) wurden in pseudonymisierter Form zur Verfügung gestellt.

Diagnosen lagen ICD-10 kodiert und für den ambulanten Sektor mit Modifikatoren vor, d. h. mit Hinweisen zur Diagnosesicherheit $(G=$ gesichert, $A$ = ausgeschlossen, $V=$ Verdacht auf, $Z=Z u$ stand nach) [26]. Für die Arzneimitteldaten wurden die Pharmazentralnummer, der ATC-Kode und die Anzahl der definierten Tagesdosen (DDD) [27] je Verordnung übermittelt. Ambulante vertragsärztliche Leistungen wurden als Leistungsziffern nach dem einheitlichen Bewertungsmaßstab (EBM) [28] übermittelt. Zu unterscheiden sind hierbei für die Kassenärztliche Bundesvereinigung und Kassenärztlichen Landesvereinigungen spezifische oder auch abgewandelte und dann mit einem Buchstabenzusatz versehene EBM-Ziffern. Für die stationären Aufenthalte lagen neben den Diagnosen auch Informationen zu Operationen und Prozeduren gemäß der amtlichen Klassifikation zum Verschlüsseln von Operationen, Prozeduren und allgemein medizinischen Maßnahmen (OPS) vor [29]. Die Leistungen der Heilmittelverordnungen konnten über die Gebührenpositionsnummer laut Heilmittelpositionsnummernkatalog identifiziert werden [30].

Da für die Operationalisierung der Indikatoren ambulante Leistungen herangezogen werden, musste geprüft werden, ob diese ggf. im Rahmen der hausarztzentrierten Versorgung (HzV) nach $\S 73 b$ SGB V pauschal vergütet werden. Für diese Leistungen liegen dann keine EBM-Ziffern vor, womit eine Indikatorenberechnung für Versicherte im $\mathrm{HzV}$ unmöglich wird. Um dies bei der Berechnung der Indikatoren zu berücksichtigen und um diese Versicherten bei 
der Indikatorenberechnung ausschließen zu können, wurden seitens der Krankenkasse für Versicherte, die im Beobachtungszeitraum Teilnehmer an der HzV waren, Beginn- und Ende-Datum der HzV-Zugehörigkeit übermittelt. Hierbei war bei wechselnder Teilnahme während des Beobachtungszeitraums auch mehr als ein Datensatz pro Versicherten möglich.

\section{Tracerpopulationen}

Basis für die Analyse diagnosebezogener Indikatoren bildeten jeweils Patientenpopulationen mit einer definierten Erkrankung. In dem hier durchgeführten Vorhaben wurden Krankheitsgruppen mit hoher Prävalenz („Volkskrankheiten“) sowie mit Präventions- und Verbesserungspotenzial durch eine integrierte Versorgung gewählt und als Tracer bezeichnet - die Population entsprechend als Tracerpopulation [24]. (Zur Übersicht siehe \Tab. 1.) Bei der Falldefinition für die kalenderjahresbezogenen Analysen wurden ambulante (vertragsärztliche) gesicherte und stationäre Aufnahme-, Neben- und Hauptentlassungsdiagnosen einbezogen. Mit wenigen Ausnahmen (s.w.u.) wurden nur intern validierte Diagnosen einbezogen [31, 32]. Als solche gelten in diesem Vorhaben stationäre Hauptentlassungsdiagnosen und ambulante gesicherte Diagnosen, die in mindestens zwei Quartalen im Kalenderjahr dokumentiert werden. Je nach Tracer bzw. Indikator (siehe Tab. A1 im Anhang) wurden verschiedene Definitionskriterien festgelegt und angewendet:

- ambulante Diagnose in mindestens einem Quartal (M1Q-Kriterium)

- ambulante Diagnose in mindestens 2 Quartalen (M2Q-Kriterium)

- mindestens eine stationäre Aufnahmediagnose (M1A-Kriterium)

- mindestens eine stationäre Hauptentlassungsdiagnose (M1E-Kriterium)

- mindestens eine stationäre Nebendiagnose (M1N-Kriterium)

Bei 16 der 19 Tracer wurde das M2Q- und/oder M1E-Kriterium angewendet. In Bezug auf den Tracer „Metabolisches Syndrom“ wurde in Abstimmung mit dem federführenden Autor des Indikatorensets festgelegt, dass mindestens drei von vier Erkrankungen (Diabetes, Hypertonie, Fettstoffwechselstörung, Adipositas) mit Einmalnennung (M1Q) vorliegen müssen, um die Kriterien der Tracerpopulation zu erfüllen. Bei den zwei Tracern „Rückenschmerzen“ und „Antibiotikatherapie“ gehörten auch Patienten mit einer Einmalnennung (M1Q) zur Tracerpopulation, um auch Patienten mit akuten Rückenschmerzen und einfachem Harnwegsinfekt einzubeziehen.

Bei neun Indikatoren wurde zur Definition diagnosebezogener Ereignisse vom M2Q/M1E-Kriterium abgesehen, da singuläre Ereignisse erhoben wurden. Hier reichte auch (teilweise neben dem M1E-Kriterium) die einmalige Nennung einer stationären Aufnahme- (M1A) oder Nebendiagnose (M1N) oder einer ambulanten Diagnose in einem Quartal (M1Q), um einen Fall zu definieren ( Tab. A2 im Anhang). Indikatoren, die sich auf eine Erkrankung mit einem bestimmten Schweregrad bezogen, wie z. B. moderate (mittelgradige) und schwere Depression, wurden mit entsprechenden ICD-Kodes operationalisiert, auch wenn dadurch die Fallzahl reduziert wurde, da im ambulanten Sektor häufig unspezifisch kodiert wird (z. B. „Depressive Episoden, nicht näher bezeichnet“).

\section{Vorgehen}

Die Zähler- und Nennerbeschreibungen der Indikatoren wurden in textliche Programmieranweisungen übersetzt. Zu den Definitionskriterien wurden die entsprechenden Ein- und Ausschlussvariablen durch ein Team mit unterschiedlichen methodischen Kompetenzen und beruflichem Kontext festgelegt ( Tab. A1, im Anhang). Zweifelsfälle hinsichtlich zu berücksichtigender Leistungen oder einzuschließender ICD-Kodes wurden mit dem federführenden Autor der Indikatorenentwicklung besprochen. Im Ergebnis wurde eine tabellarische Programmiervorlage erstellt. Jeder Indikator wurde je Kalenderjahr deskriptiv analysiert, um die Stabilität des Indikators zu erkennen und ggf. bei auffälligen Verläufen die Auswahl der Variablen und die Programmierung zu überprüfen. Besonderheiten zu den Indikatoren wurden in einem Kommentarfeld (s. Spalte Anmerkungen in > Tab. 2 und 3) aufgenommen. Studienpopulation für die Schätzung der Indikatorprävalenz - das heisst für den Anteil der Versicherten, der die Indikatorkriterien erfüllt - für die einzelnen Kalenderjahre bildeten Versicherte, die im Kalenderjahr das Kriterium durchgängig beobachtbar (ganzjährig oder ab Geburt oder bis zum Tod) mit Wohnsitz in einer Region erfüllten [25]. Je nach Indikator wurde auf bestimmte Altersgruppen eingeschränkt.

- Tab. 1 Diagnosebezug, Anzahl und Operationalisierbarkeit der Indikatoren nach Tracer/Kategorie.

\begin{tabular}{|c|c|c|c|c|}
\hline ID - Tracer/Kategorie & $\begin{array}{l}\text { Diagnose- } \\
\text { bezug }\end{array}$ & $\begin{array}{l}\text { Anzahl } \\
\text { der } \\
\text { Indikato- } \\
\text { ren } \\
\text { Gesamt }\end{array}$ & $\begin{array}{l}\text { operatio- } \\
\text { nalisierbar }\end{array}$ & $\begin{array}{l}\text { Anteil } \\
{[\%]}\end{array}$ \\
\hline $1-$ Herzinsuffizienz & $\mathrm{Ja}$ & 16 & 14 & 87,5 \\
\hline 2 - Osteoporose & $\mathrm{Ja}$ & 3 & 3 & 100,0 \\
\hline $\begin{array}{l}3 \text { - Metabolisches } \\
\text { Syndrom }\end{array}$ & $\mathrm{Ja}$ & 7 & 7 & 100,0 \\
\hline $\begin{array}{l}\text { 4- Chronisch obstruktive } \\
\text { Bronchitis }\end{array}$ & $\mathrm{Ja}$ & 6 & 5 & 83,3 \\
\hline 5 - Depression & $\mathrm{Ja}$ & 4 & 4 & 100,0 \\
\hline 6 - Rheuma & $\mathrm{Ja}$ & 3 & 3 & 100,0 \\
\hline 7 - Chronischer Schmerz & $\mathrm{Ja}$ & 5 & 5 & 100,0 \\
\hline 8 - Rückenschmerzen & $\mathrm{Ja}$ & 3 & 3 & 100,0 \\
\hline 9 - Antibiotikatherapie & $\mathrm{Ja}$ & 3 & 3 & 100,0 \\
\hline 10 - Kindervorsorge & Nein & 5 & 4 & 80,0 \\
\hline 11 - Altersspezifisch & Nein & 7 & 5 & 71,4 \\
\hline 12 - Multiple Sklerose & $\mathrm{Ja}$ & 2 & 2 & 100,0 \\
\hline $\begin{array}{l}13 \text { - Koronare } \\
\text { Herzkrankheit }\end{array}$ & $\mathrm{Ja}$ & 10 & 9 & 90,0 \\
\hline 14 - Myokardinfarkt & $\mathrm{Ja}$ & 4 & 4 & 100,0 \\
\hline 15 - Schlaganfall & $\mathrm{Ja}$ & 6 & 5 & 83,3 \\
\hline 16 - Demenz & $\mathrm{Ja}$ & 8 & 6 & 75,0 \\
\hline $\begin{array}{l}17 \text { - Arzneimittelsicher- } \\
\text { heit }\end{array}$ & Ja & 1 & 1 & 100,0 \\
\hline $\begin{array}{l}18 \text { - Früherkennung/ } \\
\text { Prävention }\end{array}$ & Nein & 5 & 5 & 100,0 \\
\hline 19 - Asthma bronchiale & $\mathrm{ja}$ & 3 & 2 & 66,7 \\
\hline Summe & & 101 & 90 & 89,1 \\
\hline
\end{tabular}


> Tab. 2 Indikatoren mit Einschränkungen der Operationalisierbarkeit.

\begin{tabular}{|c|c|c|}
\hline ID & Kurzbezeichnung & Anmerkung \\
\hline \multicolumn{3}{|c|}{1 - Herzinsuffizienz } \\
\hline 1.9 & Beratung & $\begin{array}{l}\text { Ab } 2008 \text { operationalisierbar, da EBM-Ziffer zur Abrechnung der Chronikerpauschale in } \\
2008 \text { eingeführt. }\end{array}$ \\
\hline 1.11 & $\begin{array}{l}\text { Medikamentenmonitoring/ } \\
\text { Medikationsplan }\end{array}$ & $\begin{array}{l}\text { Nur Medikamentenmonitoring abbildbar, da im Beobachtungszeitraum keine Abrech- } \\
\text { nungsziffer für das Ausstellen des Medikationsplans vorhanden. }\end{array}$ \\
\hline \multicolumn{3}{|c|}{3 - Metabolisches Syndrom } \\
\hline 3.5. & $\begin{array}{l}\text { Belastungs-EKG bei } \\
\text { Adipositas }\end{array}$ & $\begin{array}{l}\text { Indikator wurde modifiziert: Belastungs-EKG beim Hausarzt (EBM-Ziffer } 03321 \text { ist bei } \\
\text { Fachinternisten und Kardiologen nicht als separate Leistung neben den Gebührenord- } \\
\text { nungspositionen } 13250,13545,13550 \text { berechnungsfähig, da es obligater bzw. fakultati- } \\
\text { ver Leistungsinhalt dieser diagnostisch-internistischen Zusatzpauschalen ist. }\end{array}$ \\
\hline \multicolumn{3}{|c|}{$4-$ COPD } \\
\hline 4.2 & $\begin{array}{l}\text { Notfallbehandlung bei } \\
\text { DMP-Patienten COPD }\end{array}$ & Ab 2008 mit Einführung des DMP COPD operationalisierbar. \\
\hline 4.4 & Teilnahme am DMP COPD & Ab 2008 mit Einführung des DMP COPD operationalisierbar. \\
\hline \multicolumn{3}{|c|}{6 - Rheuma } \\
\hline 6.1 & $\begin{array}{l}\text { Krankheitsmonitoring/ } \\
\text { Funktionsdiagnostik }\end{array}$ & Indikator wurde auf Funktionsdiagnostik eingeschränkt. \\
\hline \multicolumn{3}{|c|}{7 - Depression } \\
\hline 7.1 & Depressionsscreening & $\begin{array}{l}\text { Ab I/2009 ist die EBM-Ziffer 35100: differentialdiagnostische Klärung psychosomatischer } \\
\text { Krankheitszustände mit EBM-Ziffer } 35110 \text { die einzig verbliebene Gesprächsleistung für } \\
\text { Hausärzte im EBM. }\end{array}$ \\
\hline 7.4 & Schmerzanalyse & $\begin{array}{l}\text { Ab } 2008 \text { mit EBM-Ziffer } 30702 \text { - Zusatzpauschale für schmerztherapeutische Versorgung } \\
\text { chronisch schmerzkranker Patienten - operationalisierbar. }\end{array}$ \\
\hline \multicolumn{3}{|c|}{10 - Kindervorsorge } \\
\hline 10.2 & HPV-Impfung & $\begin{array}{l}\text { Ab } 2007 \text { operationalisierbar, da ab diesem Zeitpunkt in den Leistungskatalog der GKV } \\
\text { aufgenommen. }\end{array}$ \\
\hline \multicolumn{3}{|c|}{16 - Demenz } \\
\hline 16.7 & $\begin{array}{l}\text { Testverfahren bei Demenz- } \\
\text { Verdacht }\end{array}$ & Ab 2008 operationalisierbar mit neu eingeführten EBM-Ziffern 03242, 16340, 21340. \\
\hline 16.8 & $\begin{array}{l}\text { Hausärztlich-geriatrisches } \\
\text { Basis-Assessment }\end{array}$ & $\begin{array}{l}\text { Die EBM-Ziffer } 03360 \text { ersetzte ab Okt. } 2013 \text { die EBM-Ziffer 03240. Für die neue EBM-Ziffer } \\
\text { ist kein zusätzlicher Leistungsnachweis erforderlich. }\end{array}$ \\
\hline \multicolumn{3}{|c|}{18 - Früherkennung/Prävention } \\
\hline 18.1 & Hautkrebs-Screening & Ab 2009 mit der EBM-Ziffer 01745 - Hautkrebs-Screening operationalisierbar. \\
\hline 18.2 & $\begin{array}{l}\text { Alle vier Impfungen MMRV } \\
\text { (11-23 Monate) }\end{array}$ & $\begin{array}{l}\text { Ab } 2008 \text { mit EBM-Ziffern } 89113 \text { (Masern), 89125/89126 (Varizellen), } 89301 \text { (3fach-Imp- } \\
\text { fung Masern, Mumps, Röteln) und 89401/89402 (4fach-Impfung Masern, Mumps, Röteln, } \\
\text { Varizellen) operationalisierbar. }\end{array}$ \\
\hline 18.3 & $\begin{array}{l}\text { Mindestens eine Impfung } \\
\text { Masern, Röteln Mumps und/ } \\
\text { oder Windpocken (11-23 } \\
\text { Monate) }\end{array}$ & $\begin{array}{l}\text { Ab } 2008 \text { mit EBM-Ziffern } 89113 \text { (Masern), 89125/89126 (Varizellen), } 89301 \text { (3-fach- } \\
\text { Impfung Masern, Mumps, Röteln) und 89401/89402 (4fach-Impfung Masern, Mumps, } \\
\text { Röteln, Varizellen) operationalisierbar. }\end{array}$ \\
\hline
\end{tabular}

\section{Ergebnisse}

\section{Übersicht zur Operationalisierbarkeit}

Tab. A1 im Anhang zeigt für jeden Indikator die gewählte Operationalisierung von Zähler und Nenner. Von den 101 konsentierten Indikatoren konnten 90 (89,1\%) mit Routinedaten abgebildet werden. Bei 11 von 19 Tracern/Kategorien lag der Anteil bei 100\%. 17 Indikatoren aus drei Kategorien (Kindervorsorge, altersspezifische Indikatoren und Früherkennung/Prävention) weisen keinen Diagnosebezug auf. > Tab. 1 zeigt eine Übersicht über die Indikatoren nach Tracer/Kategorie, Diagnosebezug und Operationalisierbarkeit.

\section{Einschränkungen in Bezug auf den Beobachtungszeitraum}

Bei 15 Indikatoren waren Vor- oder Nachbeobachtungszeiten erforderlich, so dass diese nicht für alle Jahre des Evaluationszeitraums ausgewiesen werden konnten. Dies betraf bspw. den Indikator Diabetespatienten mit neudiagnostizierter Retinopathie, bei dem ein diagnosefreies Jahr - das jeweilige Vorjahr zum Beobachtungsjahr - gefordert wurde, so dass der Indikator erst ab 2007 ausgewiesen werden konnte (s. Indikator 3.6 in Tab. A1 im Anhang). Gleiches gilt für Nachbeobachtungszeiten. Bei diesen Indikatoren konnte das Jahr 2015 nicht analysiert werden - so zum Beispiel bei dem Indikator Schlaganfallpatienten, die innerhalb eines Jahres 
- Tab. 3 Begründung für fehlende Operationalisierbarkeit.

\begin{tabular}{|c|c|c|}
\hline ID & Kurzbezeichnung & Anmerkung \\
\hline \multicolumn{3}{|c|}{$1-$ Herzinsuffizienz } \\
\hline 1.5 & Langzeit-EKG & $\begin{array}{l}\text { Das Langzeit-EKG ist bei Fachinternisten und Kardiologen nicht als separate Leistung neben den Gebühren- } \\
\text { ordnungspositionen 13250, 13545, } 13550 \text { berechnungsähig, da es obligater bzw. fakultativer Leistungsin- } \\
\text { halt dieser diagnostisch-internistischen Zusatzpauschalen ist. Eine Nichteinbeziehung dieser Gebührenord- } \\
\text { nungspositionen würde zu einer Unterschätzung, eine Einbeziehung zu einer Überschätzung des Anteils mit } \\
\text { Langzeit-EKG führen. Daher ist eine Auswertung dieses Indikators nicht möglich. }\end{array}$ \\
\hline 1.12 & $\begin{array}{l}\text { Prüfung der } \\
\text { häuslichen } \\
\text { Krankenpflege }\end{array}$ & $\begin{array}{l}\text { Zähler ab } 2008 \text { nicht operationalisierbar. Die EBM-Ziffer } 01420 \text { zur Prüfung der Notwendigkeit und } \\
\text { Koordination der häuslichen Krankenpflege ist ab } 2008 \text { in der Versichertenpauschale Kapitel } 3 \text { (Hausärztli- } \\
\text { cher Versorgungsbereich) bzw. Kapitel } 4 \text { (Versorgungsbereich der Kinder- und Jugendmedizin) enthalten und } \\
\text { kann nicht zusätzlich abgerechnet werden. Ab } 2008 \text { ist damit nur die Leistung bei Fachärzten abrechenbar } \\
\text { und dokumentiert. }\end{array}$ \\
\hline \multicolumn{3}{|c|}{4 - Chronisch obstruktive Bronchitis (COPD) } \\
\hline 4.3 & $\begin{array}{l}\text { Spirometrie zur } \\
\text { Diagnose COPD }\end{array}$ & $\begin{array}{l}\text { Die Spirometrie ist bei Fachinternisten und Pneumologen nicht als separate Leistung neben den Gebühren- } \\
\text { ordnungspositionen } 13250 \text { und } 13650 \text { berechnungsfähig, da es obligater bzw. fakultativer Leistungsinhalt } \\
\text { dieser diagnostisch-internistischen Zusatzpauschalen ist. Eine Nichteinbeziehung dieser Gebührenordnungs- } \\
\text { positionen würde zu einer Unterschätzung, eine Einbeziehung zu einer Überschätzung des Anteils mit } \\
\text { spirographischer Untersuchung führen. Daher ist eine Auswertung dieses Indikators nicht möglich. }\end{array}$ \\
\hline \multicolumn{3}{|c|}{10 - Kindervorsorge } \\
\hline 10.1 & Hörtest & $\begin{array}{l}\text { Nicht operationalisierbar, da Hörtests im Rahmen der Vorsorgeuntersuchung nach der Geburt im Kranken- } \\
\text { haus nicht als Einzelleistung in den GKV-Routinedaten erfasst sind. Im ambulanten Sektor ist seit Oktober } \\
2010 \text { eine Abrechnung über die EBM-Ziffern 01704, } 01705 \text { und } 01706 \text { möglich. Für HzV-Teilnehmer gehören } \\
\text { diese Ziffern zum HzV-Ziffernkranz und werden pauschal abgerechnet. }\end{array}$ \\
\hline \multicolumn{3}{|c|}{11 - Altersspezifisch } \\
\hline 11.2 & $\begin{array}{l}\text { Kompressions- } \\
\text { therapie }\end{array}$ & Daten zur Hilfsmittelverordnung standen nicht zur Verfügung. \\
\hline 11.7 & Fallkonferenzen & $\begin{array}{l}\text { Entfällt, da die EBM-Ziffer } 37320 \text { - Fallkonferenz gemäß Anlage } 30 \text { zum BMV-Ä erst Oktober } 2017 \text { eingeführt } \\
\text { wurde. }\end{array}$ \\
\hline \multicolumn{3}{|c|}{13 - Koronare Herzkrankheit } \\
\hline 13.7 & $\begin{array}{l}\text { Medikationsplan } \\
\text { (UAW) }\end{array}$ & $\begin{array}{l}\text { Entfällt, da die EBM-Ziffer } 01630 \text { - Zuschlag für Erstellung eines Medikationsplans erst Oktober } 2016 \\
\text { eingeführt wurde. }\end{array}$ \\
\hline \multicolumn{3}{|c|}{15 - Schlaganfall } \\
\hline 15.4 & $\begin{array}{l}\text { Diagnostik } \\
\text { Vorhofflimmern }\end{array}$ & $\begin{array}{l}\text { Das Langzeit-EKG ist bei Fachinternisten und Kardiologen nicht als separate Leistung neben den Gebühren- } \\
\text { ordnungspositionen 13250, 13545, } 13550 \text { berechnungsfähig, da es obligater bzw. fakultativer Leistungsin- } \\
\text { halt dieser diagnostisch-internistischen Zusatzpauschalen ist. Eine Nichteinbeziehung dieser Gebührenord- } \\
\text { nungspositionen würde zu einer Unterschätzung, eine Einbeziehung zu einer Überschätzung des Anteils mit } \\
\text { Langzeit-EKG führen. Daher war eine Auswertung dieses Indikators nicht möglich. }\end{array}$ \\
\hline \multicolumn{3}{|c|}{16 - Demenz } \\
\hline 16.3 & $\begin{array}{l}\text { Prüfung der } \\
\text { häuslichen } \\
\text { Krankenpflege }\end{array}$ & Siehe Indikator 1.12. \\
\hline 16.5 & $\begin{array}{l}\text { Medikationsprü- } \\
\text { fung }\end{array}$ & $\begin{array}{l}\text { Entfällt, da die EBM-Ziffer } 01630 \text { - Zuschlag für Erstellung eines Medikationsplans erst Oktober } 2016 \\
\text { eingeführt wurde. }\end{array}$ \\
\hline \multicolumn{3}{|c|}{19 - Asthma bronchiale } \\
\hline 19.3 & $\begin{array}{l}\text { Lungenfunktions- } \\
\text { prüfung }\end{array}$ & $\begin{array}{l}\text { Die Spirometrie ist bei Fachinternisten und Pneumologen nicht als separate Leistung neben den Gebühren- } \\
\text { ordnungspositionen } 13250 \text { und } 13650 \text { berechnungsfähig, da es obligater bzw. fakultativer Leistungsinhalt } \\
\text { dieser diagnostisch-internistischen Zusatzpauschalen ist. Eine Nichteinbeziehung dieser Gebührenordnungs- } \\
\text { positionen würde zu einer Unterschätzung, eine Einbeziehung zu einer Überschätzung des Anteils mit } \\
\text { spirographischer Untersuchung führen. Daher war eine Auswertung dieses Indikators nicht möglich. }\end{array}$ \\
\hline
\end{tabular}

BMV-Ä: Bundesmantelvertrag-Ärzte; EBM: Einheitlicher Bewertungsmaßstab; EKG: Elektrokardiogramm; HzV: hausarztzentrierte Versorgung; UAW:

Unerwünschte Arzneimittelwirkung.

wegen Schlaganfall wieder stationär aufgenommen werden müssen (s. Indikator 15.5 in Tab. A1, im Anhang).

\section{Eingeschränkt operationalisierbare Indikatoren}

Bei 14 der 90 mit Routinedaten abbildbaren Indikatoren gab es im Untersuchungszeitraum Einschränkungen hinsichtlich der Opera- tionalisierbarkeit. Dies betraf folgende Indikatoren und Situationen ( $\triangleright$ Tab. 2).

\section{Nicht operationalisierbare Indikatoren}

Elf der konsentierten Indikatoren konnten aus unterschiedlichen Gründen nicht operationalisiert werden. > Tab. 3 weist die Leistungen aus, die für den Indikator erforderlich gewesen wären, jedoch 
für den hier betrachteten Zeitraum (2006-2015) überwiegend aus abrechnungstechnischen Gründen nicht zur Verfügung standen. EBM-Ziffern für Fallkonferenzen und Medikationsprüfung wurden erst später in die Abrechnung aufgenommen.

\section{Relevanz der hausarztzentrierten Versorgung für die Operationalisierung}

In Baden-Württemberg können Versicherte seit 2008 an der hausarztzentrierten Versorgung ( $\mathrm{HzV}$ ) teilnehmen, was bei der Indikatorberechnung (s. o.) zu berücksichtigen ist. Bei 15 von 90 operationalisierbaren Indikatoren für neun Tracer/Kategorien musste deshalb ein Ausschluss von Versicherten, die in die HzV eingeschrieben waren, erfolgen. Tab. A3 im Anhang zeigt, welche Tracer/Kategorien betroffen waren und welche Leistungen, die zur Operationalisierung herangezogen wurden, zum HzV-Ziffernkranz gehörten.

\section{Interpretationshinweise und Prüfung auffälliger Verläufe}

Einige operationalisierbare Indikatoren bezogen sich auf die Verordnung von Arzneimitteln, die auch ohne Verordnung (z.T. in anderer Dosierung wie bei nicht steroidalen Antirheumatika oder ASS) erhältlich sind oder von denen bekannt ist, dass sie wie Benzodiazepine zu einem nicht unerheblichen Anteil auch für GKV-versicherte Patienten auf Privatrezept verordnet werden [33]. Diese Indikatoren wurden dennoch in die Analyse einbezogen, auch wenn nicht sicher ausgeschlossen werden kann, dass es hierbei regionale Unterschiede im Verordnungs- und Inanspruchnahmeverhalten geben kann.

Zur Prüfung der Operationalisierung wurden die Indikatoren deskriptiv analysiert, das heißt, es wurde für jedes Kalenderjahr der Anteil der Versicherten ausgewiesen, der die Indikatorkriterien erfüllt. Anschließend wurde die grafische Darstellung hinsichtlich auffälliger Verläufe - plötzliche Zu- oder Abnahmen der Indikatorprävalenz - gesichtet, um Hinweise auf möglicherweise falsch gewählte oder falsch programmierte Kodes (ICD, EBM, OPS, ATC) zu erhalten. Hierbei ergaben sich bei den folgenden drei Indikatoren Auffälligkeiten im zeitlichen Verlauf:

- Depressionsscreening bei Patienten mit chronischem Schmerz (Indikator 7.1, - Abb. 1a)

- HPV-Impfung (Indikator 10.2, > Abb. 1b)

- Hausärztlich-geriatrisches Basis-Assessment (Indikator 16.8, Abb. 1c)

In den Abbildungen - Abb. 1a-c werden die Ergebnisse für diese Indikatoren für die in der Evaluation herangezogenen Populationen dargestellt, d. h., für die Interventionsregion Kinzigtal (KT) und als Mittelwerte für 13 strukturähnliche Kontrollregionen (CR) in 2 Gruppen (mit und ohne Ärztenetz) sowie für eine Stichprobe aus Versicherten der AOK in Baden-Württemberg (BW) (zur Methodik und Ergebnissen der Evaluation Gesundes Kinzigtal - INTEGRAL-Studie siehe [25, 34]). Diese deskriptive Analyse erfolgte in diesem Arbeitsschritt ohne Adjustierung auf Unterschiede zwischen den Untersuchungspopulationen. Ausgewiesen wird die „Indikatorprävalenz“, d. h. jeweils der Anteil der AOK-Versicherten, die den jeweiligen Indikator erfüllten.

Beim Indikator 7.1 Chronischer Schmerz: Depressionsscreening ( $>$ Abb. 1a) zeigte sich in 2009 in allen Regionen ein deutlicher An- a

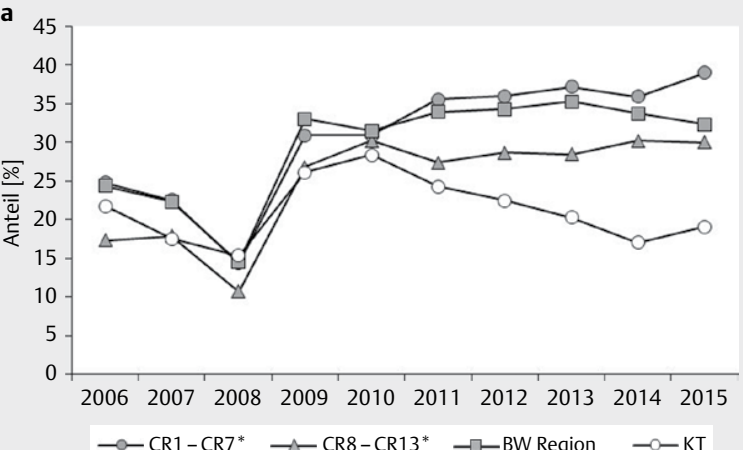

b

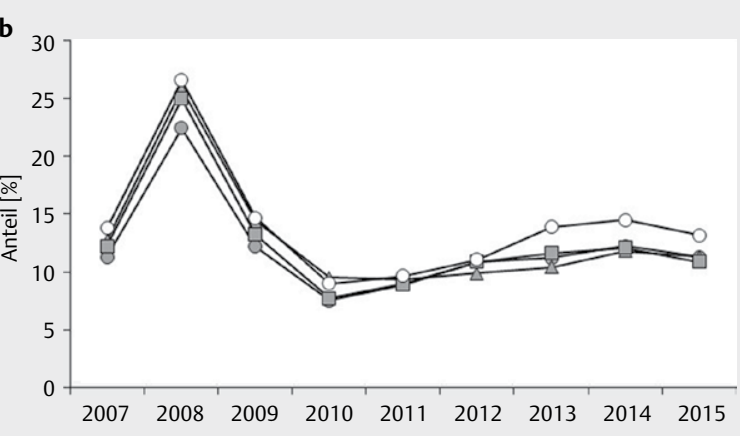

$\multimap-C R 1-C R 7^{*} \rightarrow$ CR8-CR13* $\leadsto-B W$ Region $\multimap-\mathrm{KT}$

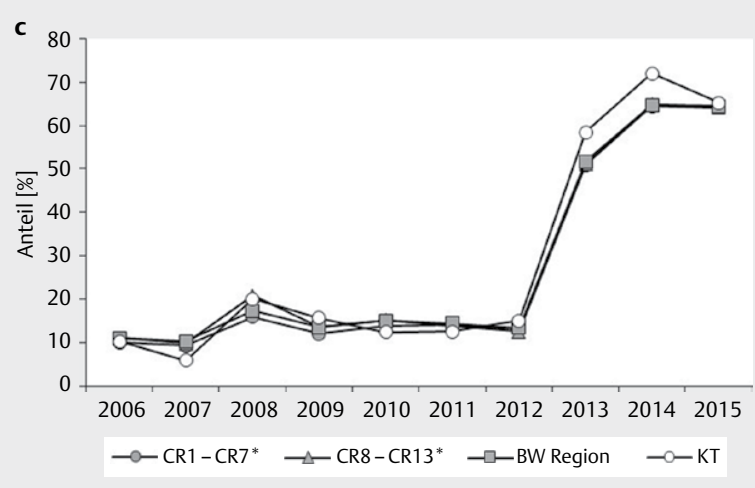

- Abb 1 a-c Hinweis: Die Abbildungen weisen eine unterschiedliche Skalierung auf CR: Kontrollregionen; * CR1-CR7 mit Arztnetz, CR8-CR13 ohne Arztnetz, BW Region: Stichprobe Baden-Württemberg, KT: Region Kinzigtal.

stieg für die Durchführung eines Depressionsscreenings. Hierzu wurde die EBM-Ziffer 35100 („Differentialdiagnostische Klärung psychosomatischer Krankheitszustände“) herangezogen. Diese war nach der Einbeziehung des hausärztlichen Gesprächs nach EBM-Ziffer 03120 in der Versichertenpauschale ab 01.01.2008 neben der EBM-Ziffer 35110 („Verbale Intervention bei psychosomatischen Krankheitszuständen“) die einzig verbliebene Gesprächsleistung für Hausärzte. In Rundschreiben wurden die Ärzte darüber informiert, dass aufgrund des starken Anstiegs in der Dokumentationshäufigkeit der Ziffer im Jahr 2009 der Verdacht bestehe, dass die Ziffer nicht entsprechend den Vorgaben dokumentiert werde und dass außerdem ein schriftlicher Vermerk über ätiologische Zusammenhänge vorliegen muss [35]. Für die Interpre- 
tation des Indikators im zeitlichen Verlauf führt diese Veränderung in den Abrechnungsmöglichkeiten zu dem Problem, dass nicht zweifelsfrei beurteilt werden kann, ob der beobachtete und nach Leitlinienempfehlung erwünschte hohe Anteil von chronischen Schmerzpatienten mit entsprechender Untersuchung auf eine korrekte Dokumentation dieser Ziffer zurückzuführen ist.

Der Verlauf des Indikators 10.2 zur HPV-Impfung ( $\$$ Abb. 1b) ist plausibel. Der starke Anstieg im ersten Jahr nach Einführung der Impfung geht auf Nachholeffekte zurück.

Bei Indikator 16.8 Demenz - Hausärztlich-geriatrisches BasisAssessment ( $\triangleright$ Abb. 1c) fällt der starke Anstieg in 2013 in allen Regionen auf. Im Beobachtungszeitraum kam es zu einem Wechsel der EBM-Ziffer. Die bis Oktober 2013 gültige EBM-Ziffer 03240 zur Abrechnung des hausärztlich-geriatrischen Basis-Assessment wurde durch die - hier berücksichtigte - EBM-Ziffer 03360 ersetzt. Für diese neue Leistungsziffer ist kein zusätzlicher Qualifikationsnachweis erforderlich, sondern sie kann bei Erfüllen des obligaten Leistungsinhaltes für die Durchführung des Basis-Assessments von jedem Hausarzt abgerechnet werden. Dies erklärt die beobachtete Zunahme im Anteil der durchgeführten Assessments (hier bei Demenzpatienten).

\section{Diskussion}

Bezogen auf die hier dargestellten 19 Tracer/Kategorien war die Umsetzung von 101 durch ein multidisziplinäres Expertenteam konsentierten Indikatoren [24] bei 90 Indikatoren - z. T. mit Anpassungen - möglich. Nur 11 Indikatoren konnten für den Beobachtungszeitraum der Studie nicht mittels der vorhandenen Kodiersysteme (hier vorrangig fehlende oder unzureichend differenzierte EBM-Kodes) abgebildet werden. Da die Routinedaten über lange Zeiträume verfügbar sind und im Vergleich zu einer Selbst- oder Fremddokumentation der Indikatoren ein ressourcensparendes Verfahren darstellen, werden sie inzwischen verschiedentlich, wie zu Beginn dargestellt, für Monitoring und Benchmarking (z. B. bei Arztnetzen) oder für Feedback-Berichte zur Fortbildung und Qualitätssicherung genutzt. Es gibt zwar eine umfangreiche Literatur zur Entwicklung und zu den Anforderungen an die Qualität von Qualitätsindikatoren [36, 37], doch wird selten die genaue Operationalisierung mit Angaben der herangezogenen oder ausgeschlossenen Kodes dargestellt. Ausnahmen sind beispielsweise das QSRVerfahren [38], das Qualitätssicherungsverfahren des IQTIG [2] und die QiSA-Indikatoren [39].

Die hier beschriebenen Indikatoren wurden für die Evaluation einer populationsbezogenen integrierten Versorgung entwickelt [25]. Das Indikatorenset erforderte deshalb den Einbezug verschiedener Sektoren und für die Evaluation einen langen Beobachtungszeitraum. Letzteres bedeutet, dass Änderungen sowohl in den Kodiersystemen als auch - wie z. B. in einigen Fällen beim EBM - in damit verbundenen Abrechnungsregelungen auftreten, die ihrerseits Einfluss auf das (Kodier-)Verhalten der Ärzte nehmen können (s.w.o. das Beispiel zu Indikator 7.1). Die visuelle Prüfung der Indikatoren über den gesamten Beobachtungszeitraum ermöglichte, solche „Strukturbrüche“ zu identifizieren und hinsichtlich möglicher Ursachen zu untersuchen. Sofern diese Auffälligkeiten nicht auf Programmierfehler oder falsche Codes zurückzuführen - und dann zu berichtigen - sind, ist je nach Fragestellung zu entscheiden, ob die betroffenen Indikatoren im Rahmen des Vorhabens berücksichtigt werden sollten. Dies gilt auch für Indikatoren, die auf Leistungen beruhen, die auch außerhalb der GKV erfolgen können, und somit zu einer Unterschätzung mittels GKV-Daten erhobenen Indikatorhäufigkeiten führen. Dies ist vor allem bei einigen Arzneimitteln (Benzodiazepine/Z-Substanzen, nicht steroidale Antirheumatika, Migränemittel, Protonenpumpenhemmer u.a.m.) der Fall, die auf Privatrezept (Benzodiazepine, Z-Substanzen) oder, z. B. in geringerer Dosierung, ohne Verordnung erhältlich sind. Auch in diesen Fällen ist je nach Fragestellung der Studie zu prüfen, ob hierdurch mögliche Verzerrungen und Fehlinterpretationen entstehen oder ob Vergleichsgruppen hiervon gleichermaßen betroffen sind.

Die deskriptive Betrachtung der Indikatoren im zeitlichen Verlauf lässt die Vermutung zu, dass bestimmte Abrechnungsziffern nicht allgemein bekannt waren (z. B. Medikamentenmonitoring) oder in einer Region erst später als in anderen in größerem Umfang eingesetzt wurden. Eine Bewertung der Versorgungsqualität ist somit immer auch abhängig von der Dokumentationsqualität. Durch Vergütungsregelungen (Incentivierung) kann Einfluss auf die Versorgungsqualität genommen werden, doch wie die dargestellten Beispiele zur differentialdiagnostischen Klärung psychosomatischer Krankheitszustände oder zum Hausärztlich-geriatrischen Basis-Assessment zeigen, sind in der Folge eine Verbesserung der Versorgung und Kodiereffekte nur schwer voneinander zu trennen.

Als Stärke der Vorgehensweise ist zunächst zu nennen, dass die Operationalisierung durch ein Team mit unterschiedlichen Kompetenzen (Medizin, Pharmazie, Versorgungsforschung) erfolgte. Ein wichtiger Schritt für die interne Qualitätssicherung stellt die systematische Überprüfung der Plausibilität der Ergebnisse zu den Indikatoren über den zeitlichen Verlauf dar. Zusätzlich wurden die Operationalisierung und adjustierten Indikatorenverläufe zusammen mit Ärzten und Ärztinnen der evaluierten Interventionsregion diskutiert, jedoch aus methodischen Gründen erst nach Projektende. Es wurden folgende Vorschläge zur Operationalisierung unterbreitet:

- Indikator 1,2 Herzinsuffizienz - Verordnung von Antiarrhythmika der Klassen I und II: Ausschluss von Patienten mit Vorhofflimmern.

- Indikator 2,3 Osteoporose - Physiotherapie nach Fraktur:

- Ausschluss von Patienten mit Demenz.

- Indikator 15,3 Schlaganfall und Verordnung von Thrombozytenaggregationshemmern: Ausschluss von Patienten mit Vorhofflimmern.

- Indikator 18,1 Hautkrebsscreening: Berücksichtigung der EBM Ziffer 01732 für die Gesundheitsuntersuchung gemeinsam mit Ziffer 01746 (für hausärztlich tätigen Arzt).

Folgende Limitationen des hier beschriebenen Vorgehens der Operationalisierung des Indikatorensets sind zu nennen: Die Angaben zur Operationalisierung und zur Operationalisierbarkeit sind an die hier betrachteten Jahre gebunden. Bei Übernahme sind die Kodes und rechtlichen Rahmenbedingungen zur Abrechnung erneut zu prüfen. Grenzen der Operationalisierbarkeit liegen in pauschalisierten Leistungen wie z. B. das Langzeit-EKG bei Fachinternisten und Kardiologen, sodass die im Indikator beschriebene Leistung nicht erfasst werden kann. Dies trifft auch auf Einzelleistungen zu, die z. B. im Rahmen der hausarztzentrierten Versorgung - oder auch 
bei Selektivverträgen - pauschal vergütet werden. Ebenso bestehen Limitationen in Bezug auf Leistungen, die nicht durch die GKV finanziert werden. An Grenzen stößt die Operationalisierung mit Routinedaten bei Indikatoren, die zur Beschreibung der Zähleroder Nennerpopulation klinische Daten oder eine Angabe des Schweregrades erfordern. Die ICD-Kodierung sieht bei einigen Erkrankungen Kodes für Schweregrade vor, doch werden diese in der ambulanten hausärztlichen Versorgung oftmals nicht verwendet. Mitunter besteht die Möglichkeit, einen Schweregrad unter Hinzuziehung weiterer Informationen zur Versorgung (Prozesse, Verordnungen, Pflege) abzubilden [40]. Auch hier ist je nach Fragestellung der Untersuchung abzuwägen, ob gegebenenfalls auf eine Einschränkung hinsichtlich des Schweregrades verzichtet werden kann, um die Fallzahl zu erhöhen. Eine weitere Einschränkung besteht bei Indikatoren, die sich auf Populationen mit Neuerkrankungen beziehen. Hierzu müssen zur Definition inzidenter Populationen Vorbeobachtungszeiten festgelegt werden. Es ist bekannt, dass bei längerer Vorbeobachtung noch prävalente Fälle identifiziert werden, die bei zu kurzen Zeiten der Population der Inzidenten zugerechnet werden $[41,42]$. In dem hier durchgeführten Projekt wurde, um nicht zu viel Nachbeobachtungszeit zu verlieren, eine erkrankungsfreie Zeit von einem Jahr festgelegt. Dabei wurde in Kauf genommen, dass einige Versicherte möglicherweise nicht inzident erkrankt waren. Da diese Festlegung sowohl die Interventionsregion als auch die Kontrollregionen betraf, wurde die Unschärfe in der Population als akzeptabel bewertet.

Die mit der Nutzung von Routinedaten verbundenen Limitationen hinsichtlich der beurteilbaren Aspekte der Versorgung sind an anderer Stelle bereits ausgeführt worden [24, 25]. Wie dargestellt, konnte ein Großteil der Indikatoren operationalisiert werden. Das heißt allerdings noch nicht, dass sich alle Indikatoren auch für kleinräumige Analysen eignen. Einige der Indikatoren (z. B. Psychotherapie bei metabolischem Syndrom oder Notfallbehandlung bei COPD) hatten geringe Nennerpopulationen oder auch geringe Fallzahlen im Zähler, so dass keine Indikatorprävalenzen ausgewiesen werden konnten.

\section{Schlussfolgerung}

Unseres Wissens wurde hier erstmalig für ein aus der Literatur entwickeltes und konsentiertes Indikatorenset zur Evaluation integrierter Versorgung die Operationalisierung so detailliert dargelegt, dass das Indikatorenset mit aktualisierten Kodes grundsätzlich zur Untersuchung von Versorgungsqualität über das vorliegende Projekt hinaus genutzt werden kann. Bei der Operationalisierung von Qualitätsindikatoren sollten Personen eingebunden werden, die mit den Kodiersystemen und Abrechnungsregelungen vertraut sind. Einen wichtigen Schritt zur internen Qualitätskontrolle der Operationalisierung stellt die Überprüfung der deskriptiven Verläufe dar. Sie ermöglicht, das Risiko von Fehlkodierungen zu minimieren. Eine transparente Darstellung der Vorgehensweise zur Operationalisierung erhöht die Akzeptanz der Ergebnisse und ermöglicht die Anschlussfähigkeit zukünftiger Vorhaben sowie die Reflexion der eigenen Vorgehensweise.

\section{Danksagung}

Das dieser Veröffentlichung zugrundeliegende Projekt „INTEGRAL - 10-Jahres-Evaluation der populationsbezogenen integrierten Versorgung Gesundes Kinzigtal in Aufbau- und Konsolidierungsphase“ wurde mit Mitteln des Innovationsausschusses beim Gemeinsamen Bundesausschuss unter dem Förderkennzeichen 01VSF16002 gefördert.

\section{Interessenkonflikt}

Die Autorinnen/Autoren geben an, dass kein Interessenkonflikt besteht.

\section{Literatur}

[1] Kleudgen S, Diel F, Burgdorf F et al. KBV entwickelt Starter-Set ambulanter Qualitätsindikatoren - AQUIK ${ }^{\circledR}$-Set. Zeitschrift für Evidenz. Fortbildung und Qualität im Gesundheitswesen 2011; 105: 54-63

[2] Institut für Qualitätssicherung und Transparenz im Gesundheitswesen (IQTIG). Qualitätsindikatoren. Available from: https://iqtig.org/ qs-instrumente/qualitaetsindikatoren/

[3] National Health Service. Quality and Outcomes Framework 2021. Available from: https://digital.nhs.uk/data-and-information/ data-tools-and-services/data-services/general-practice-data-hub/ quality-outcomes-framework-qof

[4] The National Committee for Quality Assurance (NCQA). The Healthcare Effectiveness Data and Information Set (HEDIS). Available from: https://www.ncqa.org/hedis/

[5] Agency for Healthcare Research and Quality (AHRQ). AHRQuality indicators. Available from: https://www.qualityindicators.ahrq.gov/

[6] Braun B, Müller R. Gesundheitsberichterstattung mit GKV-Daten. In: Gmünder ErsatzKasse, Rolf Müller, Bernard Braun, editors. Vom Quer- zum Längsschnitt mit GKV-Daten. Sankt Augustin: Asgard-Verl. Hippe; 2006: p 6-22

[7] Wissenschaftliches Institut der AOK (WIdO). QSR-Qualitätssicherung mit Routinedaten (Website). Available from: http://www-qualitaetssicherung-mit-routinedaten.de

[8] AOK - Die Gesundheitskasse. AOK Krankenhausnavigator (Website). Available from https://weisse-liste.krankenhaus.aok.de/

[9] Initiative Qualitätsmedizin. (Website) Available from: http://www. initiative-qualitaetsmedizin.de/

[10] Wissenschaftliches Institut der AOK (WIdO). GKV Arzneimittelindex. Available from: https://www.wido.de/forschung-projekte/arzneimittel/ gkv-arzneimittelindex/

[11] Arzneiverordnungs-Report 2020. Schwabe Ulrich, L. Wolf-Dieter, editors Heidelberg, Köln, Berlin: Springer; 2020

[12] Muijrers PE, Janknegt R, Sijbrandij J et al. Prescribing indicators. Development and validation of guideline-based prescribing indicators as an instrument to measure the variation in the prescribing behaviour of general practitioners. European journal of clinical pharmacology 2004; 60: 739-746

[13] Andres E, Bleek J, Stock J et al. Messen, Bewerten, Handeln: Qualitätsindikatoren zur Koronaren Herzkrankheit im Praxistest. Zeitschrift für Evidenz, Fortbildung und Qualität im Gesundheitswesen 2018; 137-138: 9-19 
[14] De Schreye R, Houttekier D, Deliens L et al. Developing indicators of appropriate and inappropriate end-of-life care in people with Alzheimer's disease, cancer or chronic obstructive pulmonary disease for population-level administrative databases: A RAND/UCLA appropriateness study. Palliative medicine 2017; 31: 932-945

[15] Coenen S, Ferech M, Haaijer-Ruskamp FM et al. European Surveillance of Antimicrobial Consumption (ESAC): quality indicators for outpatient antibiotic use in Europe. Quality \& safety in health care 2007; 16: 440-445

[16] Spencer R, Bell B, Avery AJ et al. Identification of an updated set of prescribing - safety indicators for GPs. The British journal of general practice : the journal of the Royal College of General Practitioners 2014; 64: e181-e190

[17] Langner I, Selke GW, Fiß T et al. Gute Beratung ist ein Gewinn. Gesundheit und Gesellschaft 2015; 7/8: 33-36

[18] Swart E, Willer C. Lässt sich die Umsetzung ärztlicher Leitlinien anhand von GKV-Routinedaten überprüfen? Gesundheitswesen 2012; 74: A125

[19] Peters F, Kreutzburg T, Kuchenbecker J et al. Behandlungsqualität in der operativ-interventionellen Gefäßmedizin - was können Routinedaten der Krankenkassen leisten? Gefässchirurgie 2020; 25: 530-540

[20] Gerlach F, Szecsenyi J. Evaluation der Hausarztzentrierten Versorgung (HzV) nach § 73b SGB V in Baden-Württemberg (2013-2016). Ergebnisbericht (Stand 0909 2014) Frankfurt und Heidelberg Verfügbar unter www aok-bw-presse de/src/php/download php. 2014.

[21] Laag S, Ullrich W, von Maydell B et al. Zwischen Kollektivsystem und Pay-for-Performance. Das BrAVo-Kennzahlensystem der BARMER GEK für Arztnetze. Gesundheitswesen aktuell 2013: p 222-247

[22] QuATRo-Projekt bringt QISA-Indikatoren in die Anwendung. Available from https://www.aok-gesundheitspartner.de/bund/qisa/praxis/ index_14315.html

[23] Donabedian A. Evaluating the quality of medical care. 1966. Milbank Q. 2005; 83: 691-729

[24] Geraedts M, Mehl C, Schmitz J et al. Development of an indicator set for the evaluation of the population-based integrated healthcare model Gesundes Kinzigtal (Healthy Kinzigtal). Zeitschrift für Evidenz, Fortbildung und Qualität im Gesundheitswesen 2020; 150: 54-64

[25] Schubert I, Stelzer D, Siegel A et al. 10-Jahres-Evaluation der populationsbezogenen integrierten Versorgung Gesundes Kinzigtal. Dtsch Arztebl Int 2021; 118: 465-472. doi:103238/arzteblm20210163 2021

[26] Deutsches Institut für medizinische Dokumentation und Information (DIMDI) im Auftrag des Bundesministeriums für Gesundheit (10. Revision). ICD 10 - GM 2019. Internationale statistische Klassifikation der Krankheiten und verwandter Gesundheitsprobleme. Available from: https://www.dimdi.de/static/de/klassifikationen/icd/icd-10-gm/ kode-suche/htmlgm2012

[27] Deutsches Institut für medizinische Dokumentation und Information (DIMDI). Anatomisch-therapeutisch-chemische Klassifikation mit Tagesdosen Köln2016. Available from: https://www.dimdi.de/ dynamic/.downloads/arzneimittel/atcddd/atc-ddd-amtlich-2016.pdf

[28] Kassenärztliche Bundesvereinigung. EBM und Gebührenordnung. 2019. Available from: http://www.daris.kbv.de/daris.asp
[29] Deutsches Institut für medizinische Dokumentation und Information (DIMDI). Operationen- und Prozedurenschlüssel (OPS) nach §301 SGB $\mathrm{V}$ - Internationale Klassifikation der Prozeduren in der Medizin. Available from: https://www.bfarm.de/DE/Kodiersysteme/Klassifikationen/OPS-ICHI/OPS/_node.html

[30] Bundeseinheitliches Positionsnummernverzeichnis für Heilmittelleistungen. Available from: https://www.gkv-datenaustausch.de/media/ dokumente/leistungserbringer_1/sonstige_leistungserbringer/ positionsnummernverzeichnisse/Heilmittel_20210401.pdf

[31] Schubert I, Köster I. Krankheitsereignis: Operationalisierung und Falldefinition. In: E. Swart, P. Ihle, H. Gothe, D. Matusiewicz, editors. Handbuch Sekundärdatenanalyse: Grundlagen, Methoden und Perspektiven. 2. vollständig überarbeitete Auflage. Bern: Hans Huber; 2014: p 358-368

[32] Schubert I, Köster I, Ihle P. Verwendung von GKV-Diagnosen in der Sekundärdatenforschung. In: E. Swart, P. Ihle, editors. Routinedaten im Gesundheitswesen. Handbuch Sekundärdatenanalyse: Grundlagen, Methoden und Perspektiven. Bern: Verlag Hans Huber; 2005: p 235-242

[33] Puteanus U, Beigi A, Rueter T et al. Benzodiazepin- und Z-Substanzverordnungen auf Kassen- und Privatrezept. Gesundheitswesen. 2016; 78: A83

[34] Schubert I, Siegel A, Graf E et al. Study protocol for a quasi-experimental claims-based study evaluating 10 -year results of the populationbased integrated healthcare model 'Gesundes Kinzigtal' (Healthy Kinzigtal): the INTEGRAL study. BMJ Open 2019; 9: e025945

[35] AAA Abrechnung aktuell. 2009. Available from: https://www.iww.de/ aaa/archiv/kassenabrechnung-psychosomatik-nach-nrn-35100-und35110-korrekt-und-nachvollziehbar-abrechnen- $\{21247$

[36] Blumenstock G. Zur Qualität von Qualitätsindikatoren. Bundesgesundheitsblatt - Gesundheitsforschung - Gesundheitsschutz 2011; 54: 154-159

[37] Geraedts M, Jäckel W, Thomeczek C et al. Qualitätsindikatoren in Deutschland. Positionspapier des Expertenkreises Qualitätsindikatoren beim Ärztlichen Zentrum für Qualität in der Medizin (ÄZQ), Berlin. Z Arztl Fortbild Qualitatssich 2005; 99: 329-331

[38] Wissenschaftliches Institut der AOK (WIdO). QSR Verfahren. Indikatorenhandbuch. Verfahrensjahr 2020. Berlin 2020. Available from: https://www.qualitaetssicherung-mit-routinedaten.de/imperia/ md/qsr/methoden/indikatorenhandbuch_2020_final.pdf

[39] QiSA. Das Indikatorensystem für die ambulante Versorgung: AOK Gesundheitskasse. 2018. Available from: http://www.aok-gesundheitspartner.de/bund/qisa/index.html

[40] Schubert I, Hammer A, Köster I. Severity assessment strategies based on administrative data using stroke as an example. Zeitschrift für Evidenz, Fortbildung und Qualität im Gesundheitswesen. 2017; 126: 66-75

[41] Abbas S, Ihle P, Köster I et al. Estimation of disease incidence in claims data dependent on the length of follow-up: a methodological approach. Health Serv Res 2012; 47: 746-755

[42] Czwikla J, Jobski K, Schink T. The impact of the lookback period and definition of confirmatory events on the identification of incident cancer cases in administrative data. BMC Medical Research Methodology $2017 ; 17: 122$ 


\section{Hinweis}

Dieser Artikel wurde gemäß des Erratums am 8. Dezember 2021 korrigiert.

\section{Erratum}

Operationalisierung von Qualitätsindikatoren mittels Routinedaten am Beispiel der Evaluation der „Integrierten Versorgung Gesundes Kinzigtal“

Köster I, Mehl C, Siegel A et al. Gesundheitswesen 2021; 83

(Suppl. 2): S87-S96

Im oben genannten Artikel wurde die geteilte Erstautorenschaft und Letztautorenschaft ergänzt und die Institutsangaben korrigiert. Richtig ist:

Ingrid Köster1* , Claudia Mehl2* , Achim Siegel3, Erika Graf4, Dominikus Stelzer4, Erik Farin-Glattacker5 Max Geraedts2**, Ingrid Schubert $1{ }^{* *}$

${ }^{*}$ geteilte Erstautorenschaft, ${ }^{* *}$ geteilte Letztautorenschaft

Die Institutsangaben lauten

1 PMV forschungsgruppe an der Medizinischen Fakultät und Uniklinik Köln, Universität zu Köln, Köln, Deutschland

2 Institut für Versorgungsforschung und Klinische Epidemiologie, Philipps-Universität Marburg, Marburg, Deutschland

3 Institut für Arbeitsmedizin, Sozialmedizin und Versorgungsforschung, Universitätsklinikum Tübingen, Tübingen, Deutschland

4 Institut für Medizinische Biometrie und Statistik, Universitätsklinikum Freiburg, Medizinische Fakultät, Albert-LudwigsUniversität Freiburg, Freiburg, Deutschland

5 Universitätsklinikum Freiburg, Institut für Medizinische Biometrie und Statistik, Sektion Versorgungsforschung und Rehabilitationsforschung, Freiburg, Deutschland

Im Abschnitt Diskussion (Seite S94, rechte Spalte) wurde der Satz

„Es ergaben sich keine Vorschläge für eine Änderung in der Operationalisierung." gelöscht und ersetzt durch:

Es wurden folgende Vorschläge zur Operationalisierung unterbreitet:

- Indikator 1.2 Herzinsuffizienz - Verordnung von Antiarrhythmika der Klassen I und II: Ausschluss von Patienten mit Vorhofflimmern.

- Indikator 2.3 Osteoporose - Physiotherapie nach Fraktur: Ausschluss von Patienten mit Demenz.

- Indikator 15.3 Schlaganfall und Verordnung von Thrombozytenaggregationshemmern: Ausschluss von Patienten mit Vorhofflimmern.

- Indikator 18.1 Hautkrebsscreening: Berücksichtigung der EBM Ziffer 01732 für die Gesundheitsuntersuchung gemeinsam mit Ziffer 01746 (für hausärztlich tätigen Arzt). 